Eur. J. Clin. Chem. Clin. Biochem.

Vol. 31, 1993, pp. 787-791

(C) 1993 Walter de Gruyter \& Co.

Berlin - New York

\title{
The Performance of the Chem-1 Revisited: An Evaluation after 3 Years
}

\author{
By B. G. Blijenberg, E. Giskes and J. D. E. van Suijlen
}

Academic Hospital Rotterdam-Dijkzigt, Department of Clinical Chemistry, Rotterdam, The Netherlands

(Received February 26/August 31, 1993)

Summary: An evaluation of the Bayer-Technicon Chem-1 multitest analyzer is described over the period 1989-1992. Various aspects like daily use, running costs and quality assessment are discussed. The whole process regarding the performance, ranging from negative feelings in the first year to satisfaction in the third, is followed critically.

\section{Introduction}

In 1988 we initiated a complete reorganization of the routine chemistry division of our laboratory. The most important reasons for this were economics, technical aging of the existing equipment and the need for cost containment, as well as streamlining of the sample handling procedures.

The existing equipment consisted of a Technicon SMAC for electrolytes and substrates and LKB reaction-rate analyzers for enzymes, together with a DuPont ACA III for emergencies $24 \mathrm{~h}$ a day and a Technicon RA-1000 for back-up.

Basic requirements were:

1. A test capacity during office hours of at least $5000-6000$ tests per day.

2. Two or three identical instruments for the whole routine test panel.

3. Emergency testing on the same instrumentation 24 hours per day.

4. Reporting time of about 30 minutes after admittance of the sample.

5. Acceptable and competitive price for the running costs.
In addition to these requirements we took into consideration: precision and accuracy aspects, technical aspects including computer facilities, reputation of the instrument and/or manufacturer, acceptability to the personnel and environmental aspects regarding the reagents.

After lengthy discussions with manufacturers as well - understandable because we are one of the largest hospital laboratories in The Netherlands - we chose the Technicon Chem-1. We were aware of the risk we were taking. As is known, the instrument was launched in 1985 in Paris with enthusiasm and success, but subsequently given up afterwards in a number of places. We finally arrived at an acceptable agreement with Bayer-Technicon, which was also based on a long-lasting relationship.

An important part of this agreement, an extensive testing of the performance of the instrument, has already been described earlier (1). This evaluation led to a positive decision regarding the purchase of two instruments with revised software. Earlier instruments had been introduced and were not accepted in other Dutch laboratories. In October 1989 our instruments were installed in the laboratory for use during office hours by 9 well-trained technicians, while in March 1990 all routine (20 chemistries) and most of the emergency tests were run at night and during the weekends as well by 40 technicians in rotating shifts. 


\section{Consideration 1990}

By the end of the year 1990 it was clear that our work-flow had improved considerably compared to the earlier situation. It was also clear that our work with both Chem-1's required much more effort sometimes than we considered acceptable and at a rather high price. We therefore decided to start a pilot study and agreed with Bayer-Technicon to discuss our experience.

The pilot study focussed on three main items:

1. Daily use of the instruments

2. Running costs

3. Quality assessment of the results

\section{Ad 1}

The main problems we met in the first year were stability, technical and reagent problems. Most of the stability problems were detected by our internal quality control system based on the Westgard rules (2), which was implemented on our local area network. The other problems were mostly clear because they were due to system or cassette malfunction.

All problems led to a waste of time because we had to recalibrate and/or restart.

Because all these problems were ad hoc we wanted, in addition to keeping the manufacturer's maintenance $\log$ book, to get a more detailed picture of the entire analytical process. We therefore introduced an internal log book for one month (November 1990), documenting every hitch and problem, the causes, the actions taken and the time delay.

Out of the 20 working days we observed only 3 passed without any problem, 10 had minor problems (delay less than 30 minutes) and 7 had more serious problems although we needed the help or advice of a BayerTechnicon service engineer only 3 times. In all situations we encountered no delay in reporting to the wards.

Over the year 1990 we had contact with the BayerTechnicon service department, by phone or in person, every two weeks on an average.

We paid special attention to the handling of emergency specimens 24 hours a day. In our earlier report we already mentioned our concern regarding the limited facility of the Chem-1 for processing these specimens. During office hours we analyze about 100 stat specimens.

In a separate study, lasting 10 days, we found a mean reporting time of 57 minutes after admittance of the sample, with a range of 31 to 85 minutes.
For the night shift the situation was different. Though all technicians were well-trained, the level of experience from person to person was different. This sometimes resulted in uncertainty, leading to more control measurements and/or recalibrations than were strictly necessary, which meant delays in reporting.

\section{Ad 2}

The offer Bayer-Technicon made regarding the running costs of the Chem-1 was favourable in comparison with other manufacturers, satisfying one of our basic requirements. On the basis of our experience in the first year it seemed without doubt that the price per test was higher than had been predicted. We tried two methods to gain insight into the average test price for the year 1990. Our hospital information system gave us the total number of reported patient requests and the counters on the systems informed us of the total number of tests. These data proved to differ by about $50 \%$, with patient requests being the lower, of course.

Evaluating all the reasons for our loss of reagents, with time-out errors of the cassettes being the most important ones, we were able to explain $30 \%$ of this difference. The remaining $20 \%$ were difficult to trace. It should be mentioned here that the test price calculated from the counter numbers of the systems agreed well with the offer Bayer-Technicon made based on our test pattern information.

\section{Ad 3}

In the internal log book mentioned in Section 1 we also noted down the reason why a sample had to be rerun for one or more requests. One reason might be technical (instrument or cassette), another some violation of our quality control rules. We also checked how many times the first result of an analysis had to be changed.

During November $19904.3 \%$ of the estimations had to be repeated, $2.0 \%$ because of technical reasons and $2.3 \%$ because of quality control reasons. The majority of these reruns were caused by creatinine and chloride, and to a lesser extent by sodium, potassium, calcium, maghesium, $\gamma$-glutamyltransferase and alanine aminotransferase assays. In only a very few cases did the first result have to be corrected, except for chloride. The figures were: 235 results out of 106992 had to be corrected, $165 \times$ chloride, $17 \times$ creatinine, $12 \times$ calcium, $12 \times$ alanine aminotransferase, $19 \times$ sodium and some other determinations only incidentally.

In nearly all situations, except where we met a cassette time-out or an instrumental fălilure, we were able to resolve all problems by recalibration. 
The external quality assessment as organized by the Dutch Quality Assessment Foundation was a reflection of our internal pattern: poor chloride and creatinine methods and sometimes warnings for sodium, potassium, calcium and $\gamma$-glutamyltransferase.

\section{Conclusion 1990}

At the end of 1990 we were balancing between giving up the systems and continuating to face the challenge. A first conclusion was that we had invested too much time and effort in our chemistry section. It was also clear, on the other hand, that we could work with both Chem-1's. From October 1989 to December 1990 we never lost control over reporting the daily routine on time.

This was in contrast with earlier experience in other Dutch laboratories, and it led to a reconfirmation of our earlier evaluation. On the other hand, it had only been possible to obtain this result thanks to the skill and dedication of our technical staff and the competence of the Bayer-Technicon service department.

Constructive discussions with Bayer-Technicon followed, which resulted in a decision to continue. We also decided to separate the handling of all emergency tests from the routine workload. This change was accomplished by June 1991. It meant a gain in reporting time of nearly half an hour.

\section{Consideration 1992}

Early in 1992 we felt the need to evaluate the whole operation again. This time for positive reasons, because we had seen a considerable improvement in our daily practice as compared with 1990 . Since the introduction of our instruments several changes had been implemented, i.e. two software updates and a modified chloride formulation, the technicians had gained more experience and they experienced less pressure in the laboratory because stat tests had been rerouted.

In order to assess this improvement objectively we considered the same aspects as described before. Once again an extensive internal log book was introduced, for the month of March 1992 and other general information was documented.

Using the same criteria as in November 1990 in the 20 days of evaluation we registered 10 without any problem, 9 with a minor delay and 1 with a delay of 60 minutes. There was no need for a service contact with Bayer-Technicon. The delays we had in this period were known to us, although we did not see them often: synchronization failure, transportation problem or quality control warning.
Over the year 1992 the service calls or visits were diminished by $50 \%$.

Looking at the running costs we also got a clearer picture. Taking into account all reagents and consumable costs we calculated an average test price that differed less than $10 \%$ from the one Bayer-Technicon had offered. During the March 1992 period we recorded that $2.8 \%$ of the estimations had to be repeated, $1.6 \%$ for technical and $1.2 \%$ for quality control reasons. This showed a considerable improvement in comparison with the previous period. No such outliers as found before were seen. We performed a total of 93188 estimations in the 20 days. Only incidentally, much less than in November 1990, did a first result have to be corrected.

\section{Additional study}

Recently a Working Group of the European Group for the Evaluation of Reagents and Analytical Systems in Laboratory Medicine published quality specifications for the imprecision and inaccuracy of analytical systems (3). It seemed to us worthwhile to compare the imprecision data of this Working Group with our earlier data, the results of a recent survey and the specifications of Bayer-Technicon.

In summary the results of a recent survey show that our Chem-1's perform less well than the quality specification of the working group expected for creatinine, sodium, chloride, calcium, magnesium, total protein and albumin. It is interesting to note that our earlier experience shows a better performance for creatinine, total protein and albumin. The specifications of the manufacturer are all fullfilled. We will report on this study more extensively later on.

\section{Discussion}

To our knowledge there is no report in the literature on a longitudinal evaluation study like ours. This is understandable because its practical value is limited. However, we felt the need to describe our experience, as never before had a new installation demanded so much attention from us in contrast with our expectations. It is clear that both we and the manufacturer have succeeded.

It was not our intention to approach mathematical exactness in our evaluation report and in the description of the various aspects we considered. In addition, we had never planned to do a study like the one described. Comparing the first year with the third we feel that we are now running reliable equipment. 
Despite this positive judgement one can ask what changes contributed most to this improvement. In our opinion there are four primary reasons.

1. As we mentioned, we started with a well-trained staff. Nevertheless, the organizational change in our laboratory, combined with the implementation of a completely new quality control system caused a dramatic change in attitude. It is thus understandable that the third year looks better than the first because of the increase in experience.

2. Technically, there were considerable improvements: newer software versions, better cassettes (drop in time-out errors by $50 \%$ ) and newer methods (e. g. for chloride).

3. We removed the emergency work from the systems, which reduced a lot of the tension in the sample handling. Nonetheless, the total workload in both years proved to be about the same.

4. Last but not least: we work with two analyzers, which means that in reality a delay is never truly a delay except in extreme situations (which occur seldomly). The question arises, of course, as to whether a stand-alone situation is attractive or not. We have no experience here, because it would have contradicted an important basic requirement we set ourselves at the outset. However, we can understand some hesitation then, although this holds true for every choice.

It might seem that we have no further critical remarks. This is not true. We may be stringent with regard to our quality control discipline but we prefer to rerun less than we really do as described before. It is difficult to compare our situation with that of other laboratories with comparable and competitive analyzers. We are aware that we have encountered problems that occur with other manufacturers as well (recall of reagents, adjustment of a calibrator value, etc.), as has been our experience.

We also feel that method improvement has not been given enough attention in the Bayer-Technicon company in relation to the Chem-1. There is a need for improvement of a number of methods. The determination of creatinine (4), sodium, potassium, chloride and calcium still demand a lot of our attention.

Finally, the question arises as to what we learned from the whole period. We feel that our choice of two Chem-1 systems was correct, although it took some time to arrive at this conviction. In a technical sense we were well supported by Bayer-Technicon. On the other hand, as we are still the only Dutch Chem-1 customer, we also sometimes feel lonely as regards support from other laboratories. The information flow from the manufacturer is meagre.

The Chem-1 was introduced too early. It is still a highly sophisticated analyzer. The implementation in our laboratory proved to be a good example of cooperation between a manufacturer well established in the field of clinical chemistry and the dedication and skill of our technical staff. There are no reasons not to include Bayer-Technicon in discussions relating the any replacement of our analyzers in the future.

\section{Acknowledgements}

The whole evaluation would not have been possible without the help of: L. J. Perret, C. J. van Leeuwen, $R$. Leeneman, $L$. T. Freimann, H. A. Roetering and G. C. Verheij.

Thanks are also due to Dr. G. J. M. Boerma and Dr. $J$. Lindemans for reading the manuscript and to $A$. P. CopperStaaner for clerical assistance.

\section{The Manufacturer's Response}

We thank the authors for the constructive and critical work they described in this evaluation report. We are pleased with this report which we recognize as a reward of all Miles-Technicon employees who worked on the Chem-1 project for several years to improve the reliability of the Chem-1 system.

The comments we have are the following:

1. More graphics would have enhanced the report. For instance by mentioning the number of service interventions between 1990, 1991 and 1992 and the number of reruns during the same period. And also by subdividing the service intervention in time ( $<30$ minutes, \pm 2 hours and $>1$ day) and kind of repair (operator or service engineer).

2. A more detailed cost calculation for reportable results is needed in order to get a clearer picture.

3. From the additional comparison study it is clear (in words) that out 20 tests the Chem-1 performs well for at least 13 tests (last survey comparison) or 16 tests (original data comparison). The specifications of the Working Group (3) are in our opinion too tight regarding sodium, chloride and calcium for every manufacturer unless they are willing to spend a lot of time and money for improvement.

4. A person who is not familiar with the system has difficulties to understand the technical terms i. e. synchronization failure, transport problem and QC warning. We recommend to explain this in more detail. 


\section{References}

1. Blijenberg, B. G., Boerma, G. J. M. \& Leijnse, B. (1989) An evaluation of the improved Technicon Chem-1 System. J. Clin. Chem. Clin. Biochem. 27, 457-469.

2. Westgard, J. O., Barry, P. I., Hunt, M. R. \& Groth, T. (1981) A multi-rule Shewhart chart for quality control in clinical chemistry. Clin. Chem. 27, 493-501.

3. Fraser, C. G., Hyltoft Petersen, P., Ricos, C. \& Haeckel, R. (1992) Proposed quality specifications for the imprecision and inaccuracy of analytical systems for clinical chemistry. Eur. J. Clin. Chem. Biochem. 30, 311-317.

4. Blijenberg, B. G., Zwang, L. \& Tilborg, M. van (1993) New approaches with the Chem-1 creatinine determination. Eur. J. Clin. Chem. Clin. Biochem. 31, 29-33.

B. G. Blijenberg, Ph. D.

University Hospital Rotterdam-Dijkzigt

Dept. Clinical Chemistry

Dr. Molewaterplein 40

NL-3015 GD Rotterdam

The Netherlands 
